\title{
Health Care Costs Associated with Addition, Titration, and Switching Antihypertensive Medications After First-Line Treatment: Results from a Commercially Insured Sample
}

\author{
Kalyani B. Sonawane Deshmukh, PhD; Jingjing Qian, PhD; Kimberly B. Garza, PharmD, MBA, PhD; \\ Bradley M. Wright, PharmD, BCPS; Peng Zeng, PhD; Cecilia M. Ganduglia Cazaban, MD, DrPH; \\ and Richard A. Hansen, PhD
}

\begin{abstract}
BACKGROUND: Treatment modifications are necessary for addressing issues related to efficacy and tolerance of first-line monotherapy, but they increase the economic burden on patients and their health plans. Understanding the differences in costs between alternative treatment modification strategies, if any, can serve as a guideline for clinical decision making and for designing cost-containment strategies.
\end{abstract}

OBJECTIVE: To compare the health care utilization costs between (a) addition (i.e., use of free-pill combinations [FPCs] or fixed-dose combinations [FDCs]) and uptitration as alternatives for addressing efficacy issues and (b) switching and downtitration as alternatives for addressing tolerance issues with first-line antihypertensive monotherapy.

METHODS: This is a retrospective cohort study that used the 2008-2012 BlueCross BlueShield of Texas claims database. Patients who had a treatment modification within 12 months of initiating antihypertensive monotherapy were identified. All-cause and disease-related health care utilization costs and drug costs were estimated from the BlueCross BlueShield health plan's perspective over a 12-month period, starting from the date of treatment modification. Propensity score-adjusted generalized linear models were used to compare costs between alternative treatment modification strategies.

RESULTS: We identified 5,998 patients who met study criteria and had a modification of treatment: $\operatorname{FPC}(n=1,395), \operatorname{FDC}(n=1,207)$, uptitration $(n=1,659)$, switching $(n=1,282)$, and downtitration $(n=455)$. All-cause and disease-related health services utilization costs were estimated for 12 months following treatment modification. Mean annual drug utilization costs were highest for the FDC strategy. All-cause inpatient and outpatient services utilization costs were significantly different between strategies used for addressing issues of tolerance and efficacy, respectively. Diseaserelated inpatient services utilization costs were lower for the FDC strategy compared with the uptitration strategy. However, disease-related inpatient services utilization costs were not significantly different for the downtitration strategy compared with the switch strategy.

CONCLUSIONS: Health care costs following treatment modifications vary by type of strategy. The high costs of FDCs may be offset by the reduction of inpatient services utilization costs. Careful consideration should be given to the differences in costs between alternative strategies.

J Manag Care Spec Pharm. 2017;23(6):691-99

Copyright $\odot 2017$, Academy of Managed Care Pharmacy. All rights reserved.

\section{What is already known about this subject}

More than $50 \%$ of newly treated hypertensive patients undergo treatment modifications, such as addition, titration, or switching within 12 months of starting antihypertensive treatment.

Studies have shown that treatment modifications increase the economic burden on patients and their health plans.

\section{What this study adds}

After comparing health care costs from competing treatment modification strategies, significant differences were found in annual health care costs.

Total annual disease-related costs were lower for the fixeddose combination (FDC) group compared with the uptitration group and lower for the downtitration group compared with the switching group.

Annual disease-related inpatients costs were lower for the FDC group compared with the uptitration group; however, inpatient costs were lower for the downtitration group compared with the switching group

H ypertension affects 1 in every 7 adults in the United States, and it is a major risk factor for cardiovascular diseases. ${ }^{1}$ The costs of health care services, antihypertensive medication, and the loss in productivity attributed to hypertension are $\$ 46$ billion each year. ${ }^{2}$ If the costs of cardiovascular diseases related to hypertension are taken in to account, the economic burden of hypertension is even higher. One of the many factors that may lead to increase in the costs of managing hypertension is treatment modification. Treatment modifications are the adjustments made to a patient's treatment regimen to address issues related to efficacy, tolerance, adherence, or satisfaction with treatment. Data from a previous study shows that health care costs for hypertensive patients receiving treatment modifications are significantly higher than for patients who do not receive treatment modifications. ${ }^{3}$

Treatment modifications are common among patients during the initial 12 months of starting hypertension treatment. ${ }^{4}$ Prevalence is particularly high among patients starting 
treatment with monotherapy, and the 2 most frequently reported reasons for treatment modifications are uncontrolled blood pressure and poor tolerance. About $50 \%$ of patients report uncontrolled blood pressure, and 30\% of patients report poor tolerance as reasons for treatment modification. ${ }^{5,6}$ While uptitration and addition of another drug are the recommended means to address efficacy-related issues, ${ }^{7}$ downtitration and switching are used to address tolerance-related issues. Regardless of the choice of treatment modification strategy, a modification of treatment will subsequently lead to changes in health care costs.

Change in the costs of drugs is an expected outcome when a patient's medication regimen is modified. In addition to drug cost, increased use of health services, particularly follow-up visits with the health care provider, are likely to increase health care expenditures. The excess economic burden resulting from treatment modifications among newly treated hypertensive patients has been reported in previous studies. ${ }^{3,8}$ However, differences in health care costs between alternative treatment modification strategies have not been compared. The purpose of this study was to compare the health care costs between the alternative strategies used for treatment modification.

\section{Methods}

A retrospective cohort study was conducted using the BlueCross BlueShield of Texas (BCBSTX) 2008-2012 commercial claims database. Enrollment data and prescription and medical claims were analyzed. A new-user design was used to identify the patient cohort. The index date of monotherapy (i.e., date of first fill) was identified for each patient. A 6-month period before the index date (starting January 1, 2008, to June 30, 2008) was used as a "wash-out" period to identify new users. Patients were included who (a) had no claim for antihypertensive drugs during the wash-out period; (b) were aged $\geq 18$ years as of their index date of monotherapy; (c) had at least 1 occurrence of hypertension diagnosis (International Classification of Diseases, Ninth Revision, Clinical Modification codes 401.xx-405.xx) for an inpatient or outpatient visit; (d) started treatment with 1 of the 5 antihypertensive drug classes (diuretics, beta blockers, calcium channel blockers [CCBs], angiotensin-converting enzyme inhibitors [ACEIs], or angiotensin-receptor blockers); and (e) were continuously enrolled during the entire observation period.

Pregnant women were excluded from the analyses. Participants covered by managed care organizations and those with prescription drug coverage through sources other than BCBSTX were excluded because their prescription claims were not available in the database. Patients who started treatment with combination therapy were excluded to minimize selection bias because these patients were more likely to have stage 2 hypertension, which could predispose them to poorer outcomes.
Patients meeting the study criteria were followed for up to 12 months to identify a treatment modification. Use and costs were then tracked for 12 months following the first treatment modification. Clinical data are not available in administrative claims databases for characterizing treatment modifications, so these were identified based on the patterns inherent in the refill histories of the participating patients. Generic Product Identifier codes, refill dates, dose, and days supply were used to identify treatment modifications. Because the addition of a drug may be prescribed in the form of free-pill combinations (FPCs) or fixeddose combinations (FDCs), analyses for the addition strategy was performed as 2 separate groups (i.e., FPC and FDC). The 5 mutually exclusive groups according to treatment modification type were as follows:

1. FPC: Patients who used combination of 2 drugs (i.e., added drugs) as 2 separate pills.

2. FDC: Patients who used combination of 2 drugs (i.e., added drugs) as a single pill.

3. Uptitration: Patients who had an increase in dose or frequency of the initial monotherapy.

4. Switch: Patients who stopped their initial antihypertensive regimen and changed to another drug within the same class or in a different drug class.

5. Downtitration: Patients who had a decrease in dose or frequency of the initial monotherapy.

All patients who had a gap of greater than 90 days in their treatment during the study period were excluded because these patients were likely to be nonpersistent. Patients who received more than 1 treatment modification during the relatively short follow-up period also were excluded because they were more likely to represent treatment-resistant hypertension. These exclusion criteria were applied to minimize selection bias.

\section{Baseline Characteristics}

Demographic characteristics, including age and sex of patients, were identified using enrollment data. Clinical characteristics included burden of comorbid conditions; existing cardiovascular conditions (e.g., ischemic heart disease, congestive heart failure, peripheral vascular diseases, and cerebrovascular diseases); and health care visits were identified from medical claims. The Charlson Comorbidity Index (CCI) was used to determine the burden of comorbid conditions. ${ }^{9}$

Antihypertensive drug class used before treatment modification (i.e., drug class of a patient's first-line monotherapy), time to treatment modification, burden of concomitant medication, and type of concomitant medications (antihyperlipidemic and antidiabetic drugs) were identified from prescription claims. All baseline characteristics were identified over a 6-month period before the start of treatment modification. The burden and type of concomitant medications were identified during the 12-month period following the treatment modification. 


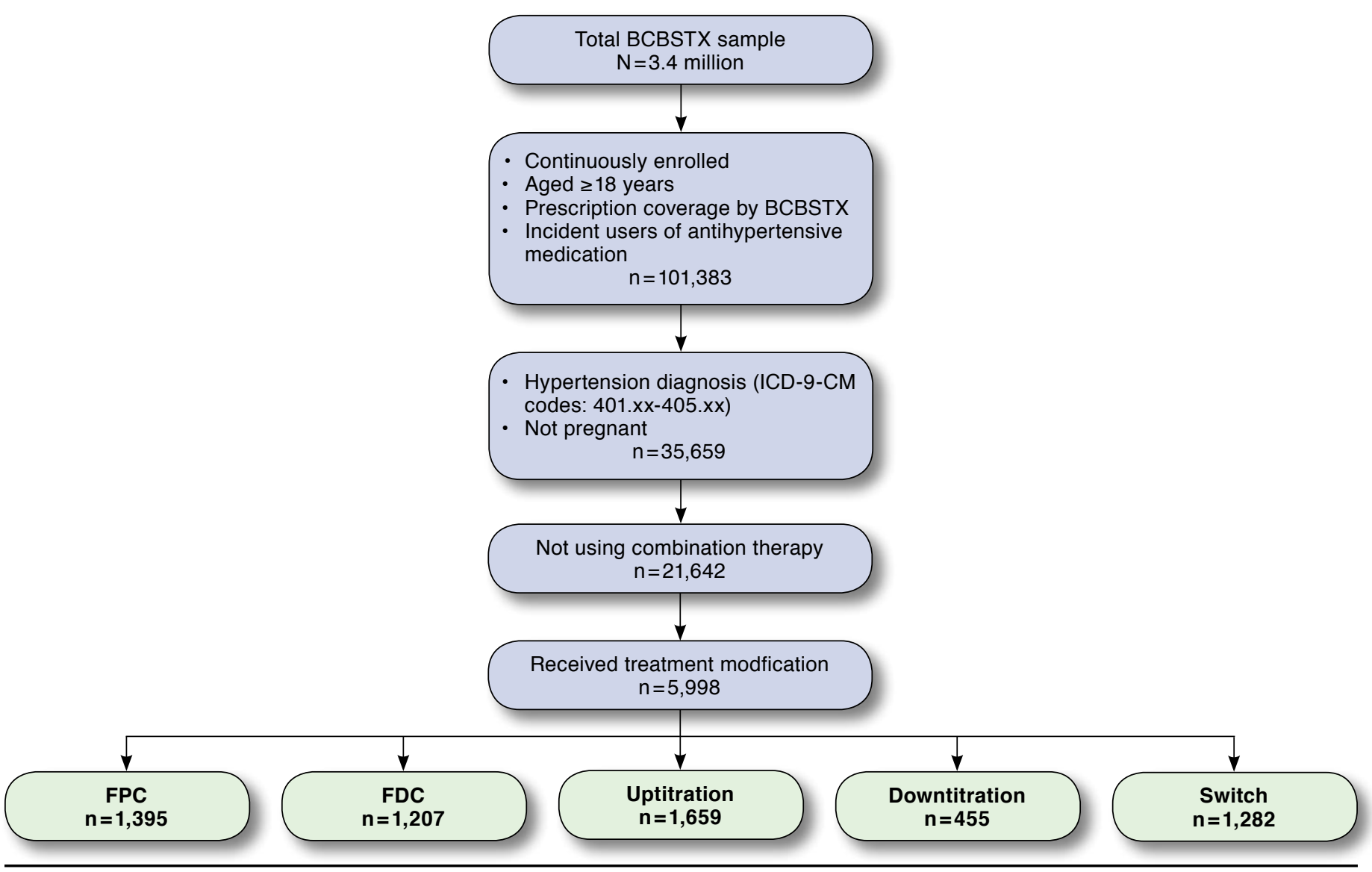

BCBSTX = BlueCross BlueShield of Texas; FDC = fixed-dose combination; FPC = free-pill combination; ICD-9-CM = International Classification of Diseases, Ninth Revision, Clinical Modification.

\section{Cost Estimation}

Health services utilization costs and antihypertensive drug costs incurred by patients were estimated over a 12-month follow-up period (starting from the treatment modification index date) and compared between the competing treatment modification strategies. Costs of using inpatient and outpatient services were estimated, along with the cost of the antihypertensive drugs paid by the health plan. In addition, total health care services utilization costs (i.e., the sum of inpatient and outpatient utilization costs) were estimated. Health services utilization costs were computed as the average cost for the overall cohort (including zero costs) and the average costs for patients using inpatient and outpatient services (non-zero costs). Allcause costs were calculated for health services claims related to any health condition. Disease-related costs were calculated for health services claims with a diagnosis of hypertension or cardiovascular conditions (a sequelae of hypertension). Costs for health care services and prescription medications were adjusted to reflect 2012 U.S. dollars using the Consumer Price Index from the U.S. Bureau of Labor Statistics. All costs were computed from the health plan's perspective (i.e., BCBSTX).

\section{Statistical Analyses}

Baseline characteristics of the 5 treatment modification groups were compared using chi-square tests and analysis of variance for categorical and continuous data, respectively. Generalized linear models were used with a gamma distribution to determine the differences in all-cause and disease-related health services utilization costs and the costs of antihypertensive drugs between every 2 competing treatment modification strategies. Costs were adjusted for differences in baseline characteristics, including age, sex, CCI, existing cardiovascular conditions, number of comedications, type of comedications, and health services utilization using 
Health Care Costs Associated with Addition, Titration, and Switching Antihypertensive

Medications After First-Line Treatment: Results from a Commercially Insured Sample

\section{TABLE 1 Baseline Characteristics of Treatment Modification Cohort by Type of Strategy}

\begin{tabular}{|c|c|c|c|c|c|c|}
\hline \multirow[b]{2}{*}{ Characteristics } & \multicolumn{6}{|c|}{ Treatment Modification Strategies } \\
\hline & $\begin{array}{c}\begin{array}{c}\text { Free-Pill } \\
\text { Combinations } \\
(\mathrm{n}=1,395)\end{array} \\
\end{array}$ & $\begin{array}{l}\text { Fixed-Dose } \\
\text { Combinations } \\
(\mathrm{n}=1,207)\end{array}$ & $\begin{array}{l}\text { Uptitration } \\
(\mathrm{n}=1,659)\end{array}$ & $\begin{array}{c}\text { Switch } \\
(\mathrm{n}=1,282)\end{array}$ & $\begin{array}{l}\text { Downtitration } \\
\quad(n=455)\end{array}$ & \multirow{2}{*}{$\frac{P \text { Value }^{\mathrm{a}}}{<0.001}$} \\
\hline \multicolumn{6}{|l|}{ Age, years } & \\
\hline $18-24$ & $(0.3)$ & $(1.6)$ & $(1.7)$ & $(1.6)$ & $(1.5)$ & \\
\hline $25-35$ & $(6.2)$ & $(8.0)$ & $(12.1)$ & $(12.6)$ & $(11.7)$ & \\
\hline $36-59$ & $1,015 \quad(72.8)$ & $(74.3)$ & $(71.8)$ & $(71.1)$ & $(71.2)$ & \\
\hline$\geq 60$ & $(20.8)$ & $(16.2)$ & $(14.4)$ & $(14.8)$ & $(15.6)$ & \\
\hline \multicolumn{6}{|l|}{ Sex } & $<0.001$ \\
\hline Male & $(58.2)$ & $(54.8)$ & $(60.0)$ & $(52.3)$ & $(56.9)$ & \\
\hline \multicolumn{6}{|l|}{ CCI score } & $<0.001$ \\
\hline 0 & $(56.4)$ & $(62.6)$ & $(64.7)$ & $(63.3)$ & $(65.9)$ & \\
\hline 1 & $374 \quad(26.8)$ & $(25.8)$ & $(23.0)$ & $(23.2)$ & $(20.4)$ & \\
\hline 2 & $(9.3)$ & $(4.6)$ & $(6.6)$ & $(7.0)$ & $(8.6)$ & \\
\hline$\geq 3$ & $(7.5)$ & $(7.1)$ & $(5.7)$ & $(6.4)$ & $(6.4)$ & \\
\hline \multicolumn{6}{|l|}{ First-line drug } & $<0.001$ \\
\hline Diuretics & $(5.7)$ & $(18.3)$ & $(5.3)$ & $(11.0)$ & $(5.5)$ & \\
\hline Beta blockers & $(8.8)$ & $(11.4)$ & $(15.7)$ & $(18.3)$ & $(17.1)$ & \\
\hline CCBs & $(28.2)$ & $(12.7)$ & $(11.8)$ & $(10.5)$ & $(8.6)$ & \\
\hline ACE inhibitors & $386 \quad(27.7)$ & $(38.2)$ & $976 \quad(58.9)$ & $(45.4)$ & $(59.3)$ & \\
\hline ARBs & $123 \quad(8.8)$ & $235 \quad(19.5)$ & $139 \quad(8.4)$ & $191 \quad(14.9)$ & $43 \quad(9.5)$ & \\
\hline Number of comedications, mean $( \pm \text { SD })^{b}$ & $4.40(2.4)$ & $4.41 \quad(2.3)$ & $4.01(2.1)$ & $4.46(2.2)$ & $4.25(2.2)$ & $<0.001$ \\
\hline \multicolumn{7}{|l|}{ Comedication type } \\
\hline Antihyperlipidemic & $(25.1)$ & $(22.2)$ & $(21.6)$ & $(20.1)$ & $(22.2)$ & $<0.001$ \\
\hline Antidiabetic & $(8.3)$ & $(7.1)$ & $(5.6)$ & $(4.6)$ & $(7.3)$ & 0.001 \\
\hline \multicolumn{7}{|l|}{ Cardiovascular diseases $^{c}$} \\
\hline Ischemic heart diseases & $(7.7)$ & $(4.0)$ & $(4.0)$ & $(4.3)$ & $(4.6)$ & $<0.001$ \\
\hline Congestive heart failure & $(1.9)$ & $(1.5)$ & $(1.6)$ & $(1.5)$ & $(1.3)$ & 0.630 \\
\hline Peripheral vascular diseases & $(1.8)$ & $(1.4)$ & $(1.3)$ & $(1.6)$ & $(1.1)$ & 0.010 \\
\hline Cerebrovascular diseases & $(3.5)$ & $(2.2)$ & $(2.3)$ & $(2.3)$ & $(3.5)$ & 0.045 \\
\hline \multicolumn{7}{|l|}{ Health care utilizationc } \\
\hline \multicolumn{6}{|l|}{ Inpatient visits } & 0.591 \\
\hline 0 & $(93.8)$ & $(95.2)$ & $(95.0)$ & $(94.8)$ & $(94.5)$ & \\
\hline $1-15$ & $(2.1)$ & $(1.8)$ & $(2.2)$ & $(1.5)$ & $(2.0)$ & \\
\hline$>15$ & $(4.1)$ & $(3.0)$ & $(2.8)$ & $(3.7)$ & $(3.5)$ & \\
\hline \multicolumn{6}{|l|}{ Outpatient visits } & $<0.001$ \\
\hline $0-3$ & $(6.5)$ & $(7.2)$ & $(10.2)$ & $(8.5)$ & $(9.2)$ & \\
\hline $4-7$ & $(6.9)$ & $(6.5)$ & $(8.0)$ & $(7.3)$ & $(8.4)$ & \\
\hline$>7$ & $1,209 \quad(86.7)$ & $(86.3)$ & $(81.9)$ & $(84.2)$ & $(82.4)$ & \\
\hline
\end{tabular}

Note: All values are $n(\%)$, unless otherwise noted.

ap values for difference between free-pill combination, fixed-dose combination, uptitration, switch, and downtitration groups.

${ }^{b}$ Measured during the follow-up period from prescription claims.

${ }^{c}$ Measured at baseline from medical claims.

$A C E=$ angiotensin-converting enzyme; $A R B s=$ angiotensin receptor blockers; $C C B s=$ calcium channel blockers; $C C I=C h a r l s o n$ Comorbidity Index; $S D=$ standard deviation .

propensity score weights. Statistical significance was tested at $P<0.05$. All analyses were conducted using SAS version 9.4 (SAS Institute, Cary, NC). The study was approved by the Auburn University Institutional Review Board.

\section{Results}

A total 5,998 patients received treatment modifications and met the study criteria: FPC $(n=1,395), \operatorname{FDC}(n=1,207)$, uptitration $(n=1,659)$, downtitration $(n=455)$, and switch ( $n=1,282$; Figure 1). The baseline characteristics of these groups are presented in Table 1.

\section{Antihypertensive Drug Costs}

The mean costs of antihypertensive drugs during the 12-month follow-up period after treatment modification are presented in Table 2. Antihypertensive drugs costs were highest for the 


\begin{tabular}{|c|c|c|c|c|}
\hline \multicolumn{5}{|c|}{$\begin{array}{ll}\text { TABLE } 2 & \text { Mean Costs and Comparison of } \\
& \text { Costs for Antihypertensive Drugs by } \\
& \text { Treatment Modification Strategy }\end{array}$} \\
\hline \multicolumn{5}{|c|}{$\begin{array}{c}\text { Drug Costs by Type of Treatment Modification Strategy (\$) } \\
\text { Mean }( \pm \text { SE) }\end{array}$} \\
\hline $\begin{array}{c}\text { Uptitration } \\
(\mathrm{n}=1,659)\end{array}$ & $\begin{array}{c}\text { Fixed-Dose } \\
\text { Combinations } \\
(\mathrm{n}=1,207)\end{array}$ & $\begin{array}{c}\text { Free-Pill } \\
\text { Combinations } \\
(\mathrm{n}=1,395)\end{array}$ & $\begin{array}{l}\text { Switch } \\
(\mathrm{n}=1,282)\end{array}$ & $\begin{array}{l}\text { Downtitration } \\
(\mathrm{n}=455)\end{array}$ \\
\hline$\$ 63(3)$ & $\$ 310(17)$ & $\$ 247(13)$ & $\$ 135(7)$ & $\$ 61(6)$ \\
\hline \multicolumn{5}{|c|}{$\begin{array}{l}\text { Note: Costs were adjusted for baseline characteristics including age, sex, CCI scores, } \\
\text { existing cardiovascular conditions, number of comedications, type of comedications, } \\
\text { and health services utilization. } \\
C C I=\text { Charlson Comorbidity Index; SE = standard error. }\end{array}$} \\
\hline
\end{tabular}

FDC group (\$310) followed by the FPC (\$247), switch (\$135), uptitration (\$63), and downtitration (\$61) groups. The mean costs were compared between alternative strategies for addressing issues of efficacy and tolerance.

Among the strategies for addressing efficacy issues, costs were significantly higher for the FDC group, compared with the uptitration and the FPC $(P<0.001)$ groups. Mean drug costs were also significantly higher for the FPC group, compared with the uptitration group $(P=0.003)$. Similarly, differences between competing strategies used to address tolerance issues (i.e., switch and downtitration) were significant $(P<0.001)$, with the switch group incurring drug costs that were on average $\$ 74$ higher than the downtitration group.

\section{All-Cause Health Services Utilization Costs}

Costs for Entire Cohort. The mean annual all-cause health services utilization costs in the treatment modification cohort were highest for the FPC group $(\$ 6,964)$, followed by downtitration $(\$ 6,595)$, FDC $(\$ 4,986)$, switch $(\$ 4,986)$, and uptitration $(\$ 4,554)$. Costs were significantly higher for patients using FPCs compared with FDCs $(P<0.001)$ and uptitration $(P<0.001$; Table 3$)$. All-cause total costs were not significantly different when compared between the uptitration and FDC groups $(P=0.16)$. Total all-cause health services utilization costs were significantly higher for the downtitration group when compared with the switch group $(P=0.004)$.

The all-cause inpatient services utilization costs were highest for the downtitration group $(\$ 2,974)$, followed by the FPC (\$1,862), FDC $(\$ 1,435)$, switch $(\$ 1,427)$, and uptitration $(\$ 1,053)$ groups. All-cause inpatient costs were significantly higher for the FPC group compared with FDC $(P=0.02)$ and uptitration $(P<0.001)$ groups. All-cause inpatient utilization costs were significantly different for the FDC group compared with the uptitration group $(P=0.005)$ and for the downtitration group compared with the switch group $(P<0.001)$. The allcause outpatient services utilization costs were $\operatorname{FPC}(\$ 5,078)$, downtitration $(\$ 3,567)$, switch $(\$ 3,562)$, FDC $(\$ 3,555)$, and uptitration $(\$ 3,515)$. All-cause outpatient costs were significantly higher for the FDC group compared with the FDC $(P<0.001)$ and uptitration groups $(P<0.001)$. Costs were not significantly different for the FDC group compared with the uptitration group and for the downtitration group compared with the switch group.

Costs for Patients Using Health Services. The mean annual all-cause total health care services utilization costs for patients using health services are presented in Table 3. Costs were highest for the FPC group $(\$ 7,188)$, followed by the downtitration $(\$ 6,827)$, FDC $(\$ 5,146)$, switch $(\$ 5,128)$, and uptitration $(\$ 4,625)$ groups. The total all-cause health care utilization costs were significantly higher for the FPC group, compared with the FDC $(P<0.001)$ and the uptitration $(P<0.001)$ groups. Costs were not significantly different for the FDC group compared with the uptitration group $(P=0.10)$. Among strategies used for addressing tolerance issues, all-cause total health services utilization costs were higher for the downtitration group, compared with the switch group $(P=0.002)$.

The mean annual all-cause health services utilization costs for patients using health services by each component are also presented in Table 3. The mean inpatient services utilization costs were highest for the downtitration group $(\$ 35,876)$, followed by the FDC $(\$ 19,120)$, uptitration, and switch $(\$ 16,034)$ groups and the FPC group $(\$ 15,790)$. Differences in all-cause inpatient services utilization costs were not significant for patients using health services to implement strategies to address efficacy issues. However, the all-cause inpatient costs were significantly higher for the downtitration strategy compared with the switch strategy $(P=0.01)$.

The outpatient health services utilization costs were highest for the FPC $(\$ 5,187)$ group, followed by the $\operatorname{FDC}(\$ 3,729)$, downtitration $(\$ 3,687)$, switch $(\$ 3,653)$, and uptitration $(\$ 3,510)$ groups. All-cause outpatient services costs were significantly higher for the FPC group compared with the FDC group $(P<0.001)$ and the uptitration group $(P<0.001)$. Costs were not significantly different for the FDC group compared with the uptitration group and for the switch group compared with the downtitration group.

\section{Disease-Related Health Services Utilization Costs}

Costs for the Entire Cohort. The total annual disease-related health care utilization costs were highest for the FPC (\$770) group, followed by the uptitration (\$412), switch (\$279), FDC (\$208), and downtitration (\$222) groups (Table 4). The total disease-related costs were significantly higher for the FPC group compared with the uptitration $(P<0.001)$ and FDC groups $(P<0.001)$. Also, costs were significantly higher for the uptitration group compared with the FDC group $(P<0.001)$. Differences in costs between the switch group and the downtitration group were statistically significant $(P=0.03)$. 
TABLE 3 Mean Cost of All-Cause Health Services Utilization During Follow-up Period by Type of Treatment Modification Strategy

\begin{tabular}{|c|c|c|c|c|c|c|c|c|c|c|}
\hline \multirow[b]{2}{*}{$\begin{array}{l}\text { Costs by Type of } \\
\text { Service Used }\end{array}$} & \multicolumn{10}{|c|}{$\begin{array}{l}\text { Costs by Type of Treatment Modification Strategy (\$) } \\
\text { Mean }( \pm \text { SE) }\end{array}$} \\
\hline & \multicolumn{2}{|c|}{ Uptitration } & \multicolumn{2}{|c|}{$\begin{array}{l}\text { Fixed-Dose } \\
\text { Combinations }\end{array}$} & \multicolumn{2}{|c|}{$\begin{array}{l}\text { Free-Pill } \\
\text { Combinations }\end{array}$} & \multicolumn{2}{|c|}{ Switch } & \multicolumn{2}{|c|}{ Downtitration } \\
\hline \multicolumn{11}{|c|}{ Health services utilization cost for the entire cohort ${ }^{\mathrm{a}}$} \\
\hline Inpatient & 1,05 & $(75)$ & 1,435 & (120) & 1,862 & (148) & 1,427 & (116) & 2,974 & (410) \\
\hline Outpatient & 3,51 & (143) & 3,555 & (168) & 5,078 & $(227)$ & 3,562 & (163) & 3,567 & $(275)$ \\
\hline Total & 4,55 & (196) & 4,998 & $(250)$ & 6,964 & (331) & 4,986 & (243) & 6,595 & $(542)$ \\
\hline \multicolumn{11}{|c|}{ Costs for patients using health services ${ }^{b}$} \\
\hline Inpatient & 16,03 & $(2,830)$ & 19,120 & $(3,720)$ & 15,790 & $(2,363)$ & 16,034 & $(2,830)$ & 35,876 & $(10,388)$ \\
\hline Outpatient & 3,51 & (141) & 3,729 & (173) & 5,187 & (225) & 3,653 & (164) & 3,687 & (277) \\
\hline Total & 4,62 & (197) & 5,146 & (254) & 7,188 & (339) & 5,128 & (245) & 6,827 & (550) \\
\hline
\end{tabular}

Note: Total health care utilization costs = inpatient utilization costs + outpatient utilization costs. Costs were adjusted for baseline characteristics including age, sex, CCI, existing cardiovascular conditions, number of comedications, type of comedications, and health services utilization.

Inpatient, outpatient, and total costs include uptitration $(n=1,659)$, fixed-dose combination $(n=1,207)$, free-pill combination $(n=1,395)$, switch $(n=1,282)$, and downtitration $(n=455)$.

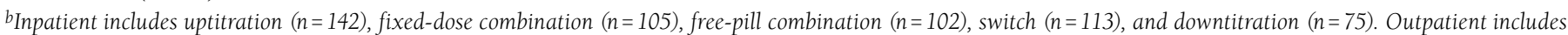
uptitration $(n=1,624)$, fixed-dose combination $(n=1,205)$, free-pill combination $(n=1,374)$, switch $(n=1,236)$, and downtitration $(n=420)$. Total includes uptitration $(n=1,624)$, fixed-dose combination $(n=1,205)$, free-pill combination $(n=1,374)$, switch $(n=1,236)$, and downtitration $(n=420)$.

$C C I=$ Charlson Comorbidity; $S E=$ standard error

Annual disease-related inpatient costs were highest for the FPC group (\$197), followed by the uptitration (\$173), switch (\$76), downtitration (\$60), and FDC (\$23) groups. Compared with the FDC group, costs were significantly higher for the FPC $(P<0.001)$ and uptitration $(P<0.001)$ groups. Diseaserelated inpatient costs were not significantly different for the FPC group compared with the uptitration group and for the downtitration group compared with the switch group. Diseaserelated outpatient costs were FPC (\$576), uptitration (\$230), switch (\$208), FDC (\$186), and downtitration (\$162). Diseaserelated outpatient costs were higher for the FPC group compared with the FDC and uptitration groups (both $P<0.001$ ), and costs were higher for the uptitration group compared with the FDC group $(P=0.002)$. Also, the difference in diseaserelated outpatient costs was statistically significant between the downtitration and switch groups $(P=0.01)$.

Costs for Patients Using Health Services. Disease-related health services utilization costs for patients using health services are presented in Table 4. The annual total diseaserelated health services utilization costs were highest for the FPC group (\$993), followed by the uptitration (\$553), switch (\$411), downtitration (\$315), and FDC (\$282) groups. Mean total disease-related health services utilization costs were significantly higher for the FPC group compared with the FDC and uptitration groups $(P<0.001)$. Costs also were significantly higher for the uptitration group compared with the FDC group $(P<0.001)$. Between the switch and downtitration groups, costs were significantly lower for the downtitration group $(P=0.01)$.
Mean annual disease-related health services utilization costs by component are also shown in Table 4. Inpatient services utilization costs were highest for uptitration $(\$ 7,692)$, followed by switch $(\$ 7,076)$, FPC $(\$ 5,746)$, downtitration $(\$ 2,985)$, and FDC $(\$ 1,731)$ groups. Disease-related inpatient services utilization costs were significantly lower for the FDC group compared with the uptitration group $(P=0.045)$. Differences between other competing strategies for addressing efficacy issues were not significant; also, differences were not significant between downtitration and switch groups. Disease-related outpatient services utilization costs were highest for FPC (\$711), followed by the uptitration (\$333), switch (\$306), FDC (\$254), and downtitration (\$238) groups. Costs were significantly higher for the FPC group compared with the FDC $(P<0.001)$ and uptitration groups $(P<0.001)$. Also, costs were significantly higher for the uptitration group compared with the FDC group $(P<0.001)$. Costs were significantly lower for the downtitration group compared with the switch group $(P<0.001)$.

\section{Discussion}

Health care costs for hypertensive patients who undergo treatment modifications are significantly higher compared with those who do not require treatment modifications. ${ }^{8}$ Alternative strategies are available for those requiring modifcations of treatment, but differences in costs between alternative TM strategies have not been published to date. In this study, costs were compared between alternatives and found that drug costs and the costs of health care visits following a treatment modification are significantly different across alternative strategies. 


\begin{tabular}{|c|c|c|c|c|c|c|c|c|}
\hline \multirow[b]{2}{*}{$\begin{array}{l}\text { Costs by Type of } \\
\text { Service Used }\end{array}$} & \multicolumn{8}{|c|}{$\begin{array}{l}\text { Costs by Type of Treatment Modification Strategy (\$) } \\
\text { Mean }( \pm \text { SE) }\end{array}$} \\
\hline & \multicolumn{2}{|c|}{ Uptitration } & \multicolumn{2}{|c|}{$\begin{array}{l}\text { Fixed-Dose } \\
\text { Combinations }\end{array}$} & Free-Pill Combinations & Switch & \multicolumn{2}{|c|}{ Downtitration } \\
\hline \multicolumn{9}{|c|}{ Health services using costs for the entire cohort ${ }^{a}$} \\
\hline Inpatient & 173 & (14) & 23 & $(2)$ & $(14)$ & $(5)$ & 60 & $(7)$ \\
\hline Outpatient & 230 & $(10)$ & 186 & $(8)$ & $(28)$ & (11) & 162 & (14) \\
\hline Total & 412 & (19) & 208 & (15) & $(40)$ & (15) & 222 & $(20)$ \\
\hline \multicolumn{9}{|c|}{ Costs for patients using health services ${ }^{b}$} \\
\hline Inpatient & 7,692 & $(3,902)$ & 1,731 & (919) & $(1,863)$ & $7,076 \quad(3,844)$ & 2,985 & $(3,260)$ \\
\hline Outpatient & 333 & (14) & 254 & (12) & (32) & $306 \quad(15)$ & 238 & (19) \\
\hline Total & 553 & $(25)$ & 282 & $(15)$ & $(50)$ & $(22)$ & 315 & $(28)$ \\
\hline \multicolumn{9}{|c|}{$\begin{array}{l}\text { Note: Total health care utilization costs =inpatient utilization costs }+ \text { outpatient utilization costs. Costs were adjusted for baseline characteristics including age, sex, CCI } \\
\text { existing cardiovascular conditions, number of comedications, type of comedications, and health services utilization. } \\
\text { a Inpatient, outpatient, and total costs include uptitration }(n=1,659) \text {, fixed-dose combination }(n=1,207) \text {, free-pill combination }(n=1,395) \text {, switch }(n=1,282) \text {, and } \\
\text { downtitration }(n=455) \text {. } \\
\text { bInpatient includes uptitration }(n=21) \text {, fixed-dose combination }(n=20) \text {, FPC }(n=26) \text {, switch }(n=20) \text {, and downtitration }(n=18) \text {. Outpatient includes uptitration } \\
(n=1,150) \text {, fixed-dose combination }(n=998) \text {, free-pill combination }(n=1,005) \text {, switch }(n=1,025) \text {, and downtitration }(n=220) \text {. Total includes uptitration }(n=1,150) \text {, } \\
\text { fixed-dose combination }(n=998) \text {, free-pill combination }(n=1,005) \text {, switch }(n=1,025) \text {, and downtitration }(n=220) \text {. } \\
C C I=\text { Charlson Comorbidity Index; SE = standard error. }\end{array}$} \\
\hline
\end{tabular}

A previous study of the U.S. Department of Defense Uniformed Services Prescription Database Project by Okano et al. (1997) estimated the costs of no change, increased dose, decrease dose, switch, and addition of drugs to ACEI and CCB initiators. ${ }^{10}$ The mean overall drug costs in this study were $\$ 60$ higher for patients who modified treatment after the initial regimen, compared with those who did not have changes in their treatment regimens. ${ }^{10}$ Another study reported an average prescription cost per step (defined as modification from initial therapy) increase from $\$ 30$ to $\$ 48$ for each additional step for patients initiating antihypertensive drugs. ${ }^{11}$

We examined whether any differences exist in drug costs for strategies used to address efficacy issues and found that patients using FPCs and FDCs had higher drug costs during the 12-month follow-up period compared with patients who uptitrated their drug dosage. This is not surprising because FDCs are typically more expensive than monotherapy drugs because of the availability of more generic versions of monotherapies, compared with branded FDCs. Also, the use of 2 drugs in combination as separate pills (FPC) is usually more expensive than monotherapy drugs for patients, as well as for the health plan. Okano et al. reported that the addition of drugs was the most expensive treatment modification, costing $\$ 167$ (34\%) more per year than initial CCB monotherapy. ${ }^{10}$

In our study, we compared uptitration, FDCs, and FPCs as alternative treatment modification strategies and found that drug costs were $\$ 63$ to $\$ 247$ higher for FDCs, compared with FPCs or a higher dose of a drug (i.e., uptitration), respectively. A recently published meta-analysis of studies compared patients using FPCs and FDCs and reported spending in excess of $\$ 70$ annually for hypertension and cardiovascular medications for the FDC group. ${ }^{12}$ The costs for FPCs also were higher compared with uptitration, with the average drug cost being $\$ 184$ higher for the FPC group.

Among strategies used for addressing tolerance issues, drug costs were $\$ 74$ higher for patients switching drugs than for patients who downtitrated their drug dosage. According to Degli Esposti et al. (2004), the annual costs of drugs for patients who continued their initial treatment was estimated to be $€ 172$ (\$220.16 in 2012 U.S. dollars), compared with €205 (\$262.40 in 2012 U.S. dollars) for those who switched to a different antihypertensive medication within the initial year of starting antihypertensive treatment. ${ }^{13}$ A direct comparison between drug costs for switching and downtitrating medications is unavailable in the current literature.

In the current study, health services utilization costs were estimated during the 12-month period following treatment modifications to better understand whether the economic burden from all-cause and disease-related health care resource utilization differs across treatment modification strategies. The mean all-cause total health services utilization costs were significantly different between treatment modification alternatives that are used to address efficacy and tolerance issues.

Costs were also compared by inpatient and outpatient services separately. Our findings, particularly pertaining to inpatient services utilization costs are important because inpatient services result in much higher costs from the perspectives of patients and their health plans. Mean annual disease-related inpatient services utilization costs were lower for the FDC group compared with the uptitration group. These results indicate 
that although the drug costs are higher for FDCs compared with uptitration, the high drug costs may be offset by reduction in disease-related inpatient services costs. In addition to the possibility of reduced disease-related inpatient utilization costs, higher efficacy, lower discontinuation rates, and better adherence also have been reported with FDCs compared with monotherapies in the literature. ${ }^{4,14,15}$ Therefore, use of FDCs may be a preferred treatment modification approach for efficacy issues with first-line antihypertensive monotherapy.

Finally, we compared health care services utilization costs between switching and downtitration (i.e., strategies for addressing tolerance issues). The association between health services utilization costs with downtitration and switching is not clearly understood. The annual disease-related inpatient services utilization costs were not significantly different for the downtitration group compared with the switch group over the 12-month follow-up period. Downtitrating dosage of an antihypertensive drug lowers its efficacy, which might increase the long-term risk of cardiovascular events. Switching may be a preferred approach for addressing tolerance issues when drug efficacy cannot be reduced.

\section{Limitations}

The results of this study should be interpreted within the context of the limitations. First, treatment modifications were identified based on patterns inherent in patient refill histories instead of on actual data for blood pressure or tolerability. It is possible that providers may have modified patient regimens for reasons not related to efficacy or tolerability of first-line monotherapy (e.g., drug costs, provider preference, or treatment dissatisfaction). Charges for health services could be different across health systems. Because this study focused on a single insurer, we assumed that contract negotiations were similar across systems; therefore, our cost estimates using paid claims were less likely to be affected by these differences in charges. This study was observational in nature with inherent limitations related to the possibility of selection bias and unobserved confounding. Finally, results of this study are not generalizable to the U.S. population, since the sample is representative only of participants covered through a commercial health insurance provider in 1 state.

\section{Conclusions}

The health services utilization costs related to modification of antihypertensive regimens vary significantly between treatment modification strategies. The high costs of FDCs may be offset by reduction in inpatient services utilization costs. Careful consideration should be given to the differences in costs between alternative strategies by health care providers when modifying patient antihypertensive regimens. From the health plan's perspective, these differences in costs will be informative for developing cost-containment strategies to minimize potential excess economic burden of treatment modifications.

\section{Authors}

KALYANI B. SONAWANE DESHMUKH, PhD, Health Services Research, Management and Policy, University of Florida, Gainesville. JINGJING QIAN, PhD, Health Outcomes Research and Policy; KIMBERLY B. GARZA, PharmD, MBA, PhD, Health Outcomes Research and Policy; PENG ZENG, PhD, Mathematics and Statistics; and RICHARD A. HANSEN, PhD, Health Outcomes Research and Policy, Auburn University, Auburn, Alabama. BRADLEY M. WRIGHT, PharmD, BCPS, Pharmacy Practice, Auburn University, Auburn, Alabama, and Internal Medicine, University of Alabama at Birmingham. CECILIA M. GANDUGLIA CAZABAN, MD, DrPH, Management, Policy and Community Health, The University of Texas Health Science Centre at Houston.

AUTHOR CORRESPONDENCE: Kalyani B. Sonawane Deshmukh, $\mathrm{PhD}$, Health Services Research, Management and Policy, University of Florida, 1225 Center Dr., HPNP 3118, Gainesville, FL 32611. Tel.: 352.273.6074; E-mail: ksonawane@phhp.ufl.edu.

\section{DISCLOSURES}

No outside funding supported this study. The dataset used in this study was created for dissertational research on the patterns and outcomes of treatment modification in hypertensive patients. Data and database support were provided by University of Texas School of Public Health/BlueCross BlueShield of Texas research program in payment systems and policy. Sonawane Deshmukh was an employee of Anthem BlueCross BlueShield from August 2015 to August 2016. Hansen has received consulting funds from Daichii Sankyo and has provided expert testimony for Allergan and Boehringer Ingelheim. All other authors have no known conflicts of interest.

Study concept and design were contributed by Sonawane Deshmukh, Garza, Wright, and Hansen. Sonawane Deshmukh and Ganduglia Cazaban collected the data, and data interpretation was performed by Sonawane Deshmukh, Qian, Wright, and Zeng. The manuscript was written primarily by Sonawane Deshmukh, along with Qian and Garza, and revised by Sonawane Deshmukh, Qian, Ganduglia Cazaban, and Hansen.

\section{REFERENCES}

1. Heidenreich PA, Trogdon JG, Khavjou OA, et al. Forecasting the future of cardiovascular disease in the United States: a policy statement from the American Heart Association. Circulation. 2011;123(8):933-44.

2. Mozaffarian D, Benjamin EJ, Go AS, et al. Heart disease and stroke statistics-2015 update: a report from the American Heart Association. Circulation. 2015;131(4):e29-322.

3. Saleh SS, Szebenyi S, Carter JA, Zacher C, Belletti D. Patterns and associated health services costs of antihypertensive drug modifications. J Clin Hypertens (Greenwich). 2008;10(1):43-50.

4. Sonawane KB, Qian J, Garza KB, et al. Patterns of treatment modifications among newly treated hypertensive patients: does choice of modification strategy affect likelihood of treatment discontinuation? J Hypertens. 2016;34(3):548-57.

5. Engel-Nitz NM, Darkow T, Lau H. Antihypertensive medication changes and blood pressure goal achievement in a managed care population. J Hum Hypertens. 2010;24(10):659-68.

6. Dusing R, Weisser B, Mengden T, Vetter H. Changes in antihypertensive therapy — the role of adverse effects and compliance. Blood Press. 1998; 7(5-6):313-15 
7. James PA, Oparil S, Carter BL, et al. 2014 evidence-based guideline for the management of high blood pressure in adults: report from the panel members appointed to the Eighth Joint National Committee (JNC 8). JAMA. 2014;311(5):507-20.

8. Payne KA, Caro JJ. Evaluating the true cost of hypertension management: evidence from actual practice. Expert Rev Pharmacoecon Outcomes Res. 2004:4(2):179-87.

9. Quan H, Sundararajan V, Halfon P, et al. Coding algorithms for defining comorbidities in ICD-9-CM and ICD-10 administrative data. Med Care. 2005;43(11):1130-39.

10. Okano GJ, Rascati KL, Wilson JP, Remund DD, Grabenstein JD, Brixner DI Patterns of antihypertensive use among patients in the US Department of Defense database initially prescribed an angiotensin-converting enzyme inhibitor or calcium channel blocker. Clin Ther. 1997;19(6):1433-45.
11. Sasane R, Brixner DI. Antihypertensive drug utilization review-a stepped approach. J Manag Care Pharm. 1997;3(3):299 [Abstract]. Available at: http://www.jmcp.org/doi/pdf/10.18553/jmcp.1997.3.issue-3.

12. Sherrill B, Halpern M, Khan S, Zhang J, Panjabi S. Single-pill vs freeequivalent combination therapies for hypertension: a meta-analysis of health care costs and adherence. J Clin Hypertens. 2011;13(12):898-909.

13. Degli Esposti L, Di Martino M, Saragoni S, et al. Pharmacoeconomics of antihypertensive drug treatment: an analysis of how long patients remain on various antihypertensive therapies. J Clin Hypertens. 2004;6(2):76-84.

14. Hilleman DE, Ryschon KL, Mohiuddin SM, Wurdeman RL. Fixed-dose combination vs monotherapy in hypertension: a meta-analysis evaluation. J Hum Hypertens. 1999;13(7):477-83.

15. Sonawane Deshmukh KB, Qian J, Garza KB, et al. Achieving adherence after first-line antihypertensive treatment: should fixed-dose combinations receive priority? J Clin Hypertens. 2016;18(9):934-41. 